\title{
Acreditación y resultados organizacionales: caso Universidad de Guayaquil
}

\author{
Fecha de recepción : 2020-01-31 • Fecha de aceptación:2020-05-15 • Fecha de publicación: 2020-09-10
}

\author{
Stephanie Delgado Estrada ${ }^{1}$ \\ Universidad de Guayaquil, Ecuador \\ Stephanie.delgadoe@ug.edu.ec \\ https://orcid.org/0000-0003-2629-1814 \\ Diana Villavicencio Chancay ${ }^{2}$ \\ Universidad de Guayaquil, Ecuador \\ diana.villavicencioc@ug.edu.ec \\ https://orcid.org/0000-0001-7895-4267 \\ Karen Hernández Ludeña ${ }^{3}$ \\ Universidad de Guayaquil, Ecuador \\ karen.hernandezl@ug.edu.ec \\ https://orcid.org/0000-0001-8533-8796
}

\section{Resumen}

Es importante conocer la calidad académica de las instituciones de educación superior a nivel nacional; por ello, el objetivo principal de esta investigación consistió en analizar el proceso de evaluación y acreditación de la Universidad de Guayaquil. En este estudio se utilizó un enfoque metodológico cualitativo, el cual permitió desarrollar la estructura y análisis de los procesos de evaluación por los que ha pasado esta institución y las categorías que ha tenido. Con la perspectiva de una revisión literaria se estructuró la teoría que sustenta el trabajo; así como los resultados más relevantes de la penúltima evaluación realizada a las Instituciones de Educación Superior del Ecuador. Entre los resultados más relevantes está la mejora en la parte académica e investigativa, pasando de una planta docente baja y producción científica nula a docentes con ingreso por concurso de méritos y mayor publicación científica y regional respectivamente. Adicional a esto, aumento de políticas y ayudas 
económicas como de acción afirmativa para que los estudiantes tengan igualdad de oportunidades en el acceso a la educación, y un incremento en las tasas de retención que permitieron continuar con sus estudios y no desertar por la falta de recursos económicos.

\title{
Palabras clave: acreditación, Universidad de Guayaquil, evaluación, educación superior.
}

\begin{abstract}
It is important to know the academic quality of higher education institutions nationwide; for this reason, the main objective of this research was to analyze the evaluation and accreditation process of the University of Guayaquil. Through the qualitative methodological approach, it allowed to develop the structure and analysis of the evaluation processes that this institution has gone through and the categories it has had. With the perspective of a literary review, the theory that supports the study work was structured; as well as the most relevant results of the penultimate evaluation made to the Higher Education Institutions of Ecuador. Among the most relevant results was the improvement in the academic and investigative part, going from a low teaching staff and zero scientific production to teachers with admission by merit competition and greater scientific and regional publication, respectively. In addition to this, increased policies and financial aid as affirmative action so that students have equal opportunities in access to education, and an increase in retention rates that allowed them to continue their studies and not drop out due to the lack of economic resources.
\end{abstract}

Keywords: accreditation, University of Guayaquil, evaluation, higher education. 


\section{Introducción}

La búsqueda de calidad en la educación superior es un propósito constante, pero asegurar esa calidad, a través de diversos medios, ha llegado a ser un verdadero desafío. Las instituciones de educación superior han determinado procesos para lograr ese aseguramiento como son la evaluación institucional y la evaluación de carrera. De la misma manera, existen bases que surgen desde la autoevaluación que realizan las instituciones y las carreras que son el punto de arranque para lograr la concordancia con lo que plantea los niveles internacionales.

Estos altos estándares internacionales se han vuelto tan necesarios que se ven reflejados en diversas áreas dentro de la educación superior, todo para lograr una acreditación, tanto desde las universidades como desde las distintas carreras al interior de las instituciones. La acreditación nacional define los puntos de partida de todos los procesos, principalmente de la enseñanza a nivel universitario.

Dentro de los estándares y derechos de los estudiantes, se indica que es un derecho el proceso de evaluación y acreditación de las carreras universitarias; a la vez indica que el órgano encargado es el Consejo de Aseguramiento de la Calidad de la Educación Superior (CEACES). Por su parte, el artículo 95 de la Ley Orgánica de Educación Superior (2012) indica que este proceso es para:

“...la mejora continua de la calidad de la educación superior y se establecerán con una vigencia de al menos tres años, período durante el cual no podrán ser modificados; consecuentemente, los procesos de acreditación considerarán únicamente criterios, estándares y las ponderaciones que hayan sido puestos en vigencia al menos tres años antes de la evaluación externa" (p.10).

De todo este proceso se desprende el análisis de las evaluaciones que hacen estas instituciones de educación superior, dando resultado al planteamiento de metas u objetivos propuestos internamente. La Universidad de Guayaquil para el año 2019, estableció en su Plan de Estratégico de Desarrollo Institucional que "la formación académica y profesional en el periodo 2016-2021, tendrá la finalidad de orientar la creación de políticas que permitan optimizar los principios de calidad, pertenencia y relevancia de las carreras de las Unidades Académicas a través del deasrrollo y fortalecimiento de la organización..."(2020, p. 53).

El sostenimiento institucional que se visualiza en una de las metas de la Universidad, se direcciona al cumplimiento de las directrices que evalúa el Organo Superior. En tal sentido, todas las instituciones de educación superior en Ecuador deben pasar por este proceso de evaluación; debido que es un derecho para sus estudiantes evidenciar la calidad académica. Por lo cual, surge el desarrollo de este trabajo de investigación posterior a las evaluaciones que ha tenido el caso de estudio.

De esta manera de estableció el siguiente objetivo: analizar el proceso de evaluación y acreditación de la Universidad de Guayaquil. Los resultados que se presenten servirán para el conocimiento y beneficio de sus estudiantes, así como sus autoridades; a la vez, se puede obtener como ejemplo para otras instituciones interesadas. 


\section{Metodología}

En esta investigación se utilizó un enfoque cualitativo a través de la recolección de información y el análisis de datos para responder al objetivo propuesto. Se visualiza una perspectiva teórica de revisión literária por el uso de revistas, libros, páginas web, ponencias y publicaciones de diferentes congresos de otras instituciones que fueron utiles para la creación de este trabajo (Hernandez Sampieri et al., 2014).

Posteriormente se detalla las evaluaciones que ha tenido el objeto de investigación y los resultados que se obtuvieron de la revisión de datos de las instituciones reguladoras. A su vez se muestra el análisis de los investigadores y los posibles resultados que puede obtener la Universidad de Guayaquil para el mejoramiento académico.

\section{La acreditación y el aseguramiento de la calidad de la educación superior}

El término calidad puede ser muy amplio en su aplicación, una definición adecuada para la calidad la señala la Organización de las Naciones Unidas para la Educación, la Ciencia y la Cultura (UNESCO): se reconoce sobre todo por el tenor de sus principios éticos y pedagógicos. La atraviesan varios conflictos y paradojas: la paradoja entre la expansión y la dispersión de la demanda, por una parte, y el paro que afecta a un número cada vez mayor de diplomados, por otra; entre el deber de igualdad y de justicia y los límites financieros de una masificación de esta forma de educación; finalmente, entre la obligación ética y moral y las diversas tentaciones de abuso de los conocimientos y los descubrimientos. Frente a estas tensiones y paradojas, la educación superior debe elaborar una nueva ambición, recurrir a su adaptabilidad, su flexibilidad y su imaginación para desarrollar capacidades de resolución de problemas y de anticipación, armarse de un espíritu crítico constante, y promover el trabajo de equipo, sin abandonar jamás su filtro ético (UNESCO, 1998, pág. 14).

Se hace necesario la conceptualización de la calidad como una construcción social, que debe ir de la mano de la definición de grupos de interés para lograr captar sus principales necesidades, tanto al interior de las unidades educativas, como en sus diversos entornos. La acreditación en instituciones de educación superior, por otra parte, nace en concordancia a la necesidad de asegurar la calidad en los procesos universitarios. Sin embargo, debe considerarse, como plantea Kumar (2011), que la acreditación no consiste en un pasaporte de garantía de la calidad en la educación superior, ya que representa una declaración pública sobre la capacidad de la institución para ofrecer programas eficaces y servicios basados en requisitos acordados.

La Universidad San Martín Porres (2020) indica, por su parte, que la acreditación universitaria es el cumplimiento de un grupo de estandares relacionados a las gestiones de procesos académicos y administrativos que se hacen en las universidades. Mientras que para el Sistema Nacional de Evaluación, Acreditación y Certificación de la Calidad Educativa de Peru (2020), estable que es un registro evaluativo para el conocimiento público y periodico en una institución, relacionados en el área de estudio, programa o planificación de una carrera profesional, basado en estándares de gestión y administración. 
La acreditación debe ser conveniente con las instituciones de educación superior, puesto que la misma no sólo debe ser capaz de cumplir su misión, sino que a la vez debe mantenerla con los programas, servicios y también el acceso a los recursos. La congruencia debe ir de la mano con los objetivos institucionales planteados y con la oferta académica de la institución de educación superior (Kumar, 2011). Dichos procesos han demostrado concientización respecto al engranaje entre educación superior y alta calidad.

Con lo expuesto, prevalece la importancia de la evaluación para cohesionar esa necesidad de la calidad en la educación superior que es innegable con el logro de una acreditación que garantice la calidad. La conformación de indicadores globales comparativos debe alinearse gracias a una metodología acorde a las necesidades, y no darse únicamente por un organismo nacional, internacional o una estructura de acuerdos entre partes (Rama, 2009).

\section{Resultados}

\section{Sistemas de acreditación de instituciones de educación superior en América latina}

En América Latina la educación superior está tomando impulsos, pese a que persisten riesgos, como también amenazas. El Times Higher Education (2017), en su Ranking de las mejores universidades latinas, ubica entre las cinco mejores a instituciones de educación superior de Brasil, Chile y Colombia, estas fueron: la Universidad Estatal de Campinas, la Universidad de São Paulo, la Pontificia Universidad Católica de Chile, la Universidad de Chile y Universidad de Los Andes, tal como se muestra en la Tabla 1.

Tabla 1.

Latin America University Rankings 2017

\begin{tabular}{llllllll}
\hline Rango & Nombre & Puntuación & Citas & $\begin{array}{l}\text { Ingreso } \\
\text { de la } \\
\text { industria }\end{array}$ & $\begin{array}{l}\text { Perspectiva } \\
\text { internacional }\end{array}$ & Investigación & Enseñando \\
\hline 1 & $\begin{array}{l}\text { Universidad Estatal } \\
\text { de Campinas, Brasil }\end{array}$ & 87,9 & 73,7 & 67,0 & 54,4 & 98,3 & 93,6 \\
\hline 2 & $\begin{array}{l}\text { Universidad de São } \\
\text { Paulo, Brasil }\end{array}$ & 87,5 & 70,5 & 50,2 & 57,5 & 100,0 & 93,9 \\
\hline 3 & $\begin{array}{l}\text { Pontificia Universidad } \\
\text { Católica de Chile, } \\
\text { Chile }\end{array}$ & 85,7 & 95,0 & 44,7 & 89,5 & 93,4 & 73,6 \\
\hline 4 & $\begin{array}{l}\text { Universidad de Chile, } \\
\text { Chile }\end{array}$ & 81,1 & 92,5 & 34,9 & 78,8 & 87,0 & 72,2 \\
\hline 5 & $\begin{array}{l}\text { Universidad de Los } \\
\text { Andes, Colombia }\end{array}$ & 77,7 & 92,9 & 54,4 & 78,0 & 79,4 & 68,4 \\
\hline
\end{tabular}

Fuente: “Latin America Univesity Rankings" por Times Higher Education 2017

En base al estudio elaborado por Times Higher Education (2017) las Universidades mejor rankeadas se encuentran en Brasil, Chile y Colombia, por lo que se tomó como base los sistemas de acreditación de estos países. En la evaluación de 13 indicadores respecto a la docencia, 
investigación, citas en publicaciones académicas, alcance internacional e ingresos del sector empresarial, despuntó Brasil con la Universidad Estatal de Campiña y la Universidad de São Paulo. En la primera se realizó la II Reunión anual de Expertos de Evaluación y Acreditación del Consejo de Evaluación y Acreditación Internacional (CEAI) (2016) y también forma parte de la Unión de Universidades de América Latina y el Caribe (UDUAL) (2017).

Los sistemas de evaluación y acreditación en los países surgen de manera necesaria para contribuir al aseguramiento de la calidad. De hecho, asociaciones como la Red Iberoamericana para el Aseguramiento de la Calidad en la Educación Superior (2017) fueron constituidas para lograr la cooperación internacional en la evaluación y acreditación de la calidad de la educación superior. Los países entendieron la necesidad de instituciones que contribuyan a asegurar dicha calidad. En la Tabla 2 se presentan los organismos de aseguramiento de la calidad al interior de Brasil, Chile y Colombia.

Tabla 2.

Entidades aseguradoras de la calidad de la Educación Superior

\begin{tabular}{llll}
\hline País & Entidad evaluadora & Año de creación & Link \\
\hline Brasil & $\begin{array}{l}\text { Sistema Nacional de Evaluación de la } \\
\text { Educación Superior (SINAES) }\end{array}$ & 2.004 & http://portal.inep.gov.br \\
\hline Chile & Comisión Nacional de Acreditación (CNA) & 2.016 & https://www.cnachile.cl \\
& & & \\
\hline Colombia & Consejo Nacional de Acreditación (CNA) & 1.992 & https://www.cna.gov.co \\
& & & \\
\end{tabular}

Fuente: SINAE, 2017, H. Congreso Nacional, Ley No. 20.129, CNA, 2017

En Brasil, el Sistema Nacional de Evaluación Superior (2017) cumple con el fortalecimiento del desarrollo y la mejora sistemática de la educación superior, integrando un modelo de evaluación cuidadoso que debe cumplir con la pertinencia y rigurosidad, pero a la vez mostrar flexibilidad debido a las características de las universidades de la región. En Chile prevalece la evaluación y acreditación a la gestión universitaria, tanto en el ámbito institucional, como el académico. Evalúa puntos básicos como la estructura organizacional, sin dejar de la mano la necesidad de asegurar la calidad en torno a la oferta académica (Comisión Nacional de Acreditación, 2017). En el caso de Colombia, el CNA evalúa aspectos como: la coherencia y pertinencia de la misión con la naturaleza, tradición, objetivos y logros institucionales; con los procesos académicos y administrativos, con los principios constitucionales y los objetivos de la educación superior (Consejo Nacional de Educación Superior, 2017). A continuación, en la Tabla 3 se presentan las principales dimensiones de evaluación. 
Tabla 3.

Dimensiones de evaluación/autoevaluación institucional

\begin{tabular}{llll}
\hline Dimensión & Brasil & Colombia & Chile \\
\hline Misión y Proyecto institucional & $\mathrm{X}$ & $\mathrm{X}$ & $\mathrm{X}$ \\
\hline Planificación, ejecución y control & $\mathrm{X}$ & $\mathrm{X}$ & $\mathrm{X}$ \\
\hline Investigación & $\mathrm{X}$ & $\mathrm{X}$ & $\mathrm{X}$ \\
\hline Proceso de enseñanza & $\mathrm{X}$ & $\mathrm{X}$ & $\mathrm{X}$ \\
\hline Posgrado & $\mathrm{X}$ & & $\mathrm{X}$ \\
\hline Pertinencia e impacto social & & $\mathrm{X}$ & $\mathrm{X}$ \\
\hline Comunicación con la sociedad & $\mathrm{X}$ & & \\
\hline Procesos académicos & $\mathrm{X}$ & $\mathrm{X}$ & $\mathrm{X}$ \\
\hline Creación artística y cultura & $\mathrm{X}$ & $\mathrm{X}$ & $\mathrm{X}$ \\
\hline Estudiantes & $\mathrm{X}$ & $\mathrm{X}$ & $\mathrm{X}$ \\
\hline Infraestructura física & $\mathrm{X}$ & $\mathrm{X}$ & $\mathrm{X}$ \\
\hline Recursos financieros & & $\mathrm{X}$ & \\
\hline Sostenibilidad financiera & $\mathrm{X}$ & & $\mathrm{X}$ \\
\hline Visibilidad nacional e internacional & & $\mathrm{X}$ & $\mathrm{X}$ \\
\hline Evaluación/Autoevaluación & $\mathrm{X}$ & $\mathrm{X}$ & \\
\hline
\end{tabular}

Fuente: elaboración propia basada en el Sistema Nacional de Evaluación de la Acreditación, 2017; Reglamento sobre áreas de acreditación 2013 por Comisión Nacional de Acreditación, 2017; Acuerdo 03-2014 Lineamientos para la acreditación internacional por Consejo Nacional de Educación Superior, 2017.

\section{Sistemas de acreditación en el Ecuador - aseguramiento de la calidad}

Según el Consejo Nacional de Evaluación y Acreditación UNESCO / IESALC (2013) desde la década de los 90 en el país se ha venido diseñando sistemas de evaluación y acreditación con la finalidad de que se garantice la calidad y la igualdad de oportunidades en las instituciones de educación superior, tal y como se muestra en la Figura 1, a continuación: 


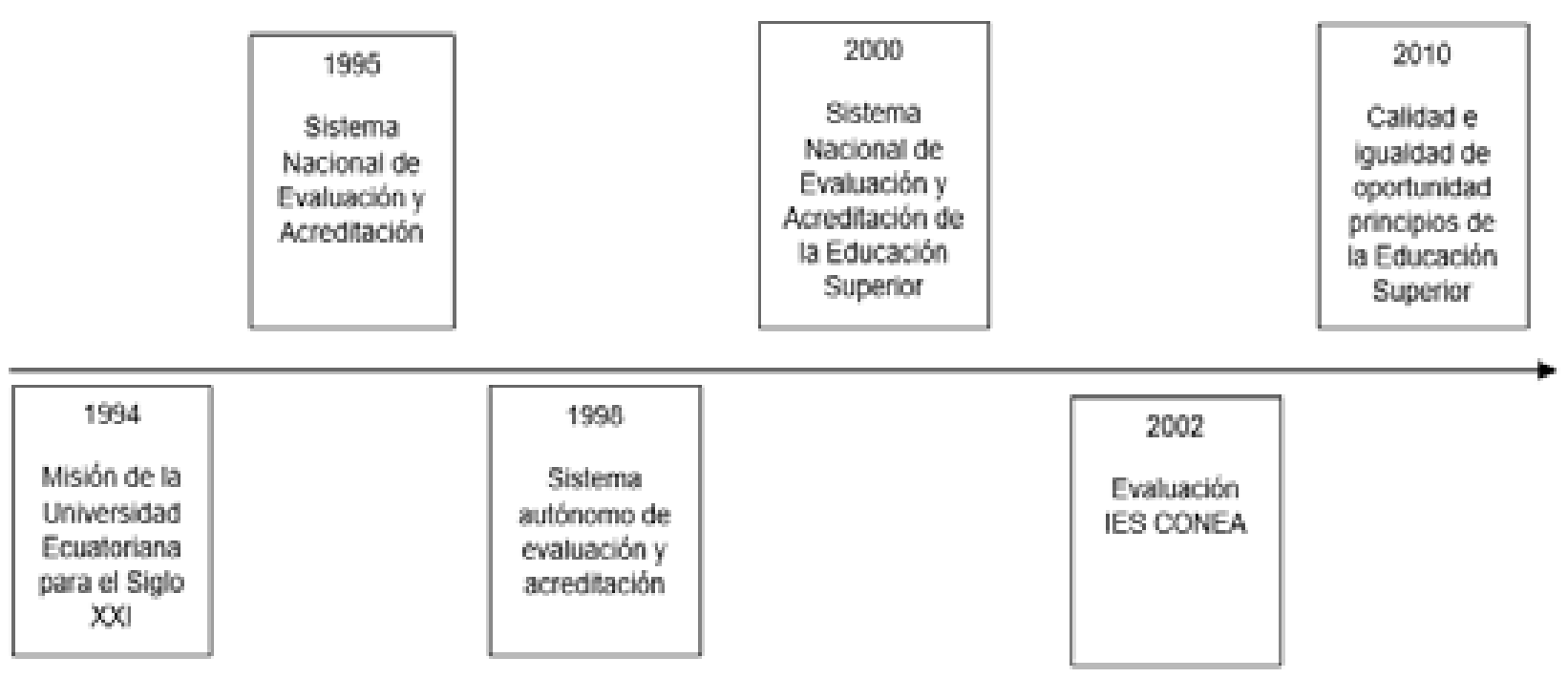

Figura 1. Cronología de las visiones de calidad en el Ecuador.

Fuente: elaboración propia basada en Digital Observatory for Higher Education in Latin America and The Caribbean

Desde el 2011 y hasta la actualidad, la institución que evalúa que se cumpla con los estándares de calidad en la educación superior ecuatoriana es el Consejo de Evaluación, Acreditación y Aseguramiento de la calidad de la Educación Superior (CEAACES), este establece que los procesos de evaluación con fines de acreditación sean quinquenales, y que las IES se categoricen de acuerdo a la Tabla 4.

Tabla 4.

Categorías Instituciones de Educación Superior

\begin{tabular}{ll}
\hline Categorías & Resultado \\
\hline A , B , C & $\begin{array}{l}\text { Institución que ha aprobado satisfactoriamente la evaluación y han obtenido la } \\
\text { acreditación con vigencia quinquenal. }\end{array}$ \\
\hline D & $\begin{array}{l}\text { Institución en proceso de acreditación, posteriormente podrán acreditarse en una nueva } \\
\text { evaluación. }\end{array}$ \\
\hline
\end{tabular}

Fuente: CEAACES (2013)

Los procesos de evaluación se basan en el "Modelo de Evaluación Institucional de Universidades y Escuelas Politécnicas". Este modelo considera seis criterios que están relacionados con las funciones sustantivas de las universidades y escuelas politécnicas: organización, academia, investigación, vinculación con la sociedad, recursos e infraestructura y estudiantes, de estos el que mayor peso tiene en el proceso de evaluación es la academia con el $36 \%$, y el de menor es vinculación con el 3\%.

En el año 2009, 2013 y 2016 se realizaron procesos de evaluación en el Ecuador, de los cuales se obtuvieron los resultados que se reflejan en la Tabla 5: 
Tabla 5.

Número de universidades según categoría

\begin{tabular}{llll}
\hline Categoría / Año & 2009 & 2013 & 2016 \\
\hline $\mathrm{A}$ & 11 & 3 & 8 \\
\hline $\mathrm{B}$ & 9 & 18 & 28 \\
\hline $\mathrm{C}$ & 13 & 14 & 19 \\
\hline $\mathrm{D}$ & 9 & 6 & $-\cdot-----$ \\
\hline $\mathrm{E}$ & 26 & $-\cdots---$ & $-\cdot----$ \\
\hline Total & 68 & 41 & 55 \\
\hline
\end{tabular}

Fuente: elaboración propia basada en Informes de evaluación de las IES.

La Universidad de Guayaquil es una de las más grandes del país, la misma que en los procesos de evaluación y acreditación realizados, obtuvo la categoría "B" en el año 2009 y la categoría "D" en el 2013, tomando como referencia esta última categoría, este resultado implicaba para la universidad que fuera intervenida, por lo que el Consejo de Educación Superior (CES) dispuso mediante resolución que una Comisión Interventora cubra de forma integral todos los aspectos de la gestión universitaria, con la intención de que esta analice todos los procesos que se daban en la institución, apuntando a la mejora para que en un futuro proceso de evaluación la Universidad se eleve de categoría.

La Comisión de Intervención y Fortalecimiento Institucional (s.f.) elaboró el Plan de excelencia Universitaria de la Universidad de Guayaquil, en este contemplaba diversos aspectos que le permitan fortalecer dos ejes principales:

- Solucionar los problemas que originaron las causales de la intervención

- Fortalecer la institución en base a los actuales estándares de calidad de la Educación Superior.

Basados en los ejes propuestos en el Plan de Fortalecimiento la Universidad de Guayaquil, trabajó alineando sus procesos a los criterios institucionales, para que estos se mejoren, y como resultado de ese plan, se obtuvieron las siguientes mejoras y con ello la Universidad de Guayaquil obtuvo la categoría "B": Para el 2016 se elaboró una Planificación Estratégica de Desarrollo Institucional (PEDI) que guio todas las actividades y procesos de la institución, apuntando a obtener objetivos estratégicos que guíen el cumplimiento de la acreditación. La Planificación Operativa Anual (POA) de la Universidad para el 2016, se articuló al PEDI. Tanto el PEDI y el POA, tuvo involucramiento en su elaboración de las autoridades pertinentes.

La institución cuenta con un modelo educativo ecológico. En lo que respecta a la docencia, la Secretaría de Educación Superior, Ciencia, Tecnología e Innovación (Senescyt), indica en sus registros que, en el 2016 el 90\% de la planta docente tiene maestría y PHD en comparación al 2013 que solo era el $37 \%$, de igual manera el número de docentes a tiempo completo pasó de 105 (2013) a 1152 (2016). Este incremento en el número de docentes, específicamente los titulares, se ha dado en su mayoría a través del ingreso por Concurso de Méritos y Oposición. 
Para el 2015 la Universidad comenzó a llevar los procesos de evaluación docente de mejor manera y aunque no era oficial, sirvió de base para diseñar un sistema oficial. Se evidencia mayor participación de las mujeres en el campo de la docencia. En la parte científica hubo un avance significativo, de acuerdo a las declaraciones a la prensa por parte de la Secretaría de Educación Superior, Ciencia, Tecnología e Innovación (Senescyt), se han registrado alrededor de 169 publicaciones científicas en el 2016, en comparación al 2013, que no había ninguna.

En el plano estudiantil, los alumnos cuentan con políticas que permiten que se les dé un mayor acceso a beneficios como ayudas económicas, lo que apunta a que los estudiantes no deserten de la carrera por faltas de recursos.

Por los puntos expuestos anteriormente, en la evaluación y categorización del 2016 la Universidad de Guayaquil obtuvo la Categoría B, lo que significó un gran avance para la institución, lo que implicaba que ya no estaría intervenida y que podría desarrollar sus procesos con autonomía.

\section{Retos futuros de la Universidad de Guayaquil}

Entre los retos que se le presentan a la Universidad de Guayaquil en el tema de evaluación y acreditación está el de continuar dirigiendo todos sus procesos acordes a los parámetros del "Modelo de Evaluación Institucional" y trabajar en conjunto con cada una de las carreras de esta institución, y apuntar a conseguir la Categoría "A".

Adicional a esto, apuntar a que todas las carreras acrediten, y de esta manera continuar con la oferta académica acorde a la pertinencia que tienen en la sociedad. Por esto, es importante explicar que en el Ecuador también se evalúan y se da una categoría a las carreras, esto se encuentra definido en el Reglamento de Evaluación, Acreditación y Categorización de carreras de las Instituciones de Educación Superior y en su artículo 7 señala que hay 2 componentes para este proceso: la evaluación del entorno de aprendizaje y el examen nacional de evaluación de carreras.

El primero mide según el mencionado reglamento las condiciones académicas, investigativas de gestión y organización necesarias para el desarrollo de las carreras en las instituciones de educación superior, y el segundo se centra en los conocimientos que los estudiantes hayan adquirido en su carrera durante el proceso de formación. Por lo que las carreras también guían sus procesos de acuerdo a lo que señala un modelo de evaluación establecido por el CEAACES (2014).

A nivel de universidad y de carrera, tanto los docentes, como los estudiantes, tienen una participación importante para el logro de los objetivos institucionales, y tal como se señala en los objetivos estratégicos del PEDI, la Universidad debe ejecutar los siguientes aspectos relevantes:

\section{Academia:}

- Desarrollar una oferta académica de calidad y pertinente con la demanda actual.

- Que los docentes continúen con su formación de posgrado, apuntando a obtener el doctorado,

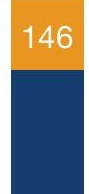


de esta manera un gran porcentaje de la planta docente tenga ese título académico.

- Que los docentes apunten a publicar en revistas de alto impacto, lo que implicaría un incremento en la producción científica y para ello continuar con las ayudas económicas que se les brindan a los docentes para que puedan asistir a eventos internacionales y con ello mejorar su producción intelectual.

Estudiantes:

- Que los estudiantes cumplan sus procesos de graduación en el tiempo establecido por la ley.

- Que los estudiantes logren desarrollarse en el campo profesional para el cual se prepararon, y para ello las autoridades competentes continuar con el seguimiento oportuno y adecuado a los graduados, e incluso mejorar la oferta académica basados en los resultados de este seguimiento.

- Que los estudiantes continúen sus estudios en la carrera institucional que han elegido y con ello aumentar la tasa de retención, a través de la promoción de las ayudas económicas.

- Que los estudiantes participen en proyectos estudiantiles académicos y científicos que los preparen para su profesión y los conviertan en constantes investigadores con la intención de apuntar a la solución de los problemas de la sociedad.

\section{Conclusiones}

Los procesos de evaluación y acreditación se han dado en las instituciones de educación superior con el objetivo de que se logre la cohesión con el aseguramiento de la calidad. Sin embargo, las dimensiones de estudio y los indicadores deben fundamentarse en principios que nazcan desde el reconocimiento del entorno donde se da la demanda académica. También, y aún más importante, la necesidad de la adaptabilidad al entorno.

Los indicadores más altos que, si bien garantizarán resultados de los productos enviados al mercado, no sólo delimitan el sendero que deben continuar las universidades y centros de educación superior. Brasil, Colombia y Chile, líderes en el ranking, demuestran la necesidad del enfoque en indicadores claves como planificación, investigación, enseñanza, pertinencia, comunicación con la sociedad.

La Universidad de Guayaquil ha apuntado a desarrollar sus procesos basados en los estándares diseñados en el Modelo de Evaluación Institucional, y está realizando cambios significativos que permitan que la institución alcance una categoría superior, y que todas sus carreras acrediten.

El proceso de evaluación en la universidad le permitió aumentar la competitividad, y acaparar más demanda, dado que se encuentra en una categoría superior, lo que le permite ser más atractiva para las personas que quieren desarrollar una carrera profesional en esta institución. 


\section{Referencias}

Asamblea Nacional. (2012). Ley Orgánica de Educación Superior . Quito: Asamblea Nacional.

CEAACES. (2013). Reglamento para la determinación de resultados del proceso de evaluación, acreditación y categorización de las Universidades y Escuelas Politécnicas y de su situación académica e institucional. http:// www.ceaaces.gob.ec/sitio/wp-content/uploads/2014/02/RESOLUCIO\%CC\%81N-No.-001-071-CEAACES-2013..-Reglamento-para-la-determinacio\%CC\%81n-de-resultados-del-Proceso-de-evaluacio\%CC\%81n-de-las-Uni1.pdf

CEAACES. (2014). Reglamento de Evaluación y Acreditación y Categorización de carreras de las Instituciones de Educación Superior.

Comisión de Intervención y Fortalecimiento Institucional. (s.f.). http://www.ces.gob.ec/index.php?option=com phocadownload \& view=category \&id=205\& $\mathbf{I t e m i d}=553$

Comisión Nacional de Acreditación. (1 de Agosto de 2017). Comisión Nacional de Acreditación. https://www. cnachile.cl/Documentos\%20de\%20Paginas/RES-DJ-01.pdf

Consejo Nacional de Educación Superior. (26 de Julio de 2017). Consejo Nacional de Educación Superior. https://www.cna.gov.co/1741/articles186359 Acuerdo 32014 Lin Acr IES.pdf

Consejo Nacional de Evaluación y Acreditación de la Educación Superior del Ecuador. (2009). Evaluación de desempeño institucional de las Universidades y Escuelas Politécnicas del Ecuador. Quito.

Consejo Nacional de Evaluación y Acreditación UNESCO/IESALC. (2003). Los antecedentes, situación actual y perspectivas de la Evaluación y la Acreditación de la Educación Superior en Ecuador. (D. O. Caribbean, Ed.) http://unesdoc.unesco.org/images/0014/001404/140478s.pdf

H. Congreso Nacional. (1 de Agosto de 2017). Ley No. 20.129. https://www.cnachile.cl/SiteAssets/Paginas/ Ley\%2020129/LEY-20129 17-NOV-2006 \%20ASEGURAMIENTO\%20DE\%20LA\%20CALIDAD\%20 (002).pdf

Hernandez Sampieri , R., Fernandez Collado , C., \& Baptista Lucio , M. (2014). Metodología de la Investigación (Sexta ed.). México, México: Mcgraw-Hill - Interamericana de editores S.A. http://observatorio.epacartagena.gov.co/wp-content/uploads/2017/08/metodologia-de-la-investigacion-sexta-edicion.compressed.pdf

Kumar, A. (2011). Calidad y acreditacion en la educación superior en Centroamérica. Revista Centroamericana de Administración Pública.

Rama, C. (2009). El nacimiento de la acreditación Internacional. Primer Congreso Internacional de Evaluación y Acreditación (págs. 291-311). Campeche: Consejo de Acreditación de la enseñanza en Contaduría y 


\section{Administración CACECA .}

Red Iberoamericana para el Aseguramiento de la Calidad en la Educación Superior . (1 de Agosto de 2017). RIACES.ORG.

SINAE. (26 de Julio de 2017). Sistema Nacional de Evaluación de la Educación. http://portal.inep.gov.br/sinaes

Sistema Nacional de Evaluación, Acreditación y Certificación de la Calidad Educativa. (2020). SINEACE. https:// www.sineace.gob.pe/acreditacion/

Times Higher Education. (26 de Julio de 2017). Times Higher Education. https://www.timeshighereducation.com/ world-university-rankings/2017/latin-america-university-rankings\#!/page/0/length/25/sort by/rank/sort order/asc/cols/stats

UDUAL. (26 de Julio de 2017). Unión de Universidades de América Latina y el Caribe. https://www.udual.org/ evaluacion.html

UNESCO. (1998). La educación superior en el siglo XXI. Conferencia Mundial de la Educación Superior (pág. 14). París: UNESCO.

Universidad de Guayaquil. (2020). Plan Estratégico de Desarrollo Institucional. Guayaquil, Ecuador: Universidad de Guayaquil. http://www.ug.edu.ec/secretaria-general-r/normativa/vigente//PLAN\%20ESTRATEGICO\%20DE\%20DESARROLLO\%20INSTITUCIONAL\%202016-2021.pdf

Universidad San Martín Porres. (2020). Facultad de Ciencias Administrativas y Recursos Humanos. https:// www.administracion.usmp.edu.pe/ice/actividades-nivel-interno/acreditacion-universitaria/ 
Copyright (c) 2020 Stephanie Marcela Delgado Estrada, Diana Villavicencio Chancay y Karen Hernández Ludeña

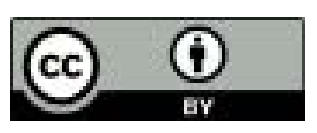

Este texto está protegido bajo una licencia internacional Creative Commons 4.0.

Usted es libre para Compartir-copiar y redistribuir el material en cualquier medio o formato

- y Adaptar el documento - remezclar, transformar y crear a partir del material-para cualquier propósito, incluso para fines comerciales, siempre que cumpla las condiciones de Atribución. Usted debe dar crédito a la obra original de manera adecuada, proporcionar un enlace a la licencia, e indicar si se han realizado cambios. Puede hacerlo en cualquier forma razonable, pero no de forma tal que sugiera que tiene el apoyo del licenciante o lo recibe por el uso que hace de la obra.

\section{$\underline{\text { Resumen de licencia - Texto completo de la licencia }}$}

